\title{
The Peritonitis as a Casuistic Clinical Manifestation of the New Coronavirus Disease SARS-CoV-2 COVID-19
}

\author{
Gorolyuk Anna Yuriyivna \\ Department of Surgery, Hospital № 7, Kriviy Rih, Ukraine \\ Email address: \\ a.gorolyuk@i.ua
}

\section{To cite this article:}

Gorolyuk Anna Yuriyivna. The Peritonitis as a Casuistic Clinical Manifestation of the New Coronavirus Disease SARS-CoV-2 COVID-19. Clinical Medicine Research. Vol. 10, No. 5, 2021, pp. 169-172. doi: 10.11648/j.cmr.20211005.13

Received: October 3, 2021; Accepted: October 21, 2021; Published: October 30, 2021

\begin{abstract}
Since the announcement of the COVID-19 pandemic (March 11, 2020), the focus of the study of complications of this new coronavirus disease has been on pneumonia and acute respiratory distress syndrome. Meanwhile, with COVID-19, acute abdominal surgical diseases develop, presumably due to the tropism of the COVID-19 virus to angiotensin-converting enzyme receptors in the digestive tract. In principle, previously known coronaviruses could cause infectious peritonitis, however, such cases of coronavirus peritonitis were observed exclusively in animals. In particular, FIPV - coronavirus is the direct cause of feline infectious peritonitis. This article describes a rare, alarming case of serous fibrinous peritonitis as a casuistic manifestation of a new coronavirus infection COVID-19 directly in humans: a 55-year-old female patient. Surgical diseases that could cause the development of this peritonitis in this patient were excluded. The purpose of the article is to point out the possibility of the development of this surgical disease, as a direct complication of the new coronavirus infection COVID-19. The article also presents the author's attempt to explain in general terms the pathogenesis of the development of peritonitis in COVID-19 in humans. Observing the second year of the pandemic, extremely rare mentions in medical scientific articles of cases of primary peritonitis in COVID-19, we can conclude that this complication is not typical in this new infection. However, given the too short period since the emergence of a new coronavirus infection COVID-19 (less than two years), as well as the ability of a new strain of coronavirus to mutate, we can assume that, in principle, the possibility of developing coronavirus peritonitis in humans is not excluded in the future.
\end{abstract}

Keywords: Coronavirus Disease SARS-CoV-2 COVID-19, Peritonitis, Serous Fibrinous Peritonitis

\section{Introduction}

Among the clinical manifestations of coronavirus disease COVID-19, caused by the previously unknown betacoronavirus SARS-CoV-2 [1, 2], the development of serous peritonitis is extremely rare. Looking at the available sources of information (scientific English, German, Ukrainian and Russian journals and scientific Internet messages), we can say that since the proclamation of the pandemic of the new coronavirus COVID-19, only several cases of peritonitis have been reported as a direct consequence of coronavirus infection. SARS-CoV-2. Some cases were described in English-language scientific journals, the other two were recorded in the territory Russian Federation [3-6]. The development of peritonitis in these patients was not directly related to acute surgical pathology of the abdominal cavity. Mechanisms of abdominal pain in SARS-CoV-2 infection can be divided into pulmonary (with abnormalities confirmed on a computed tomography), due to the lesion lower lungs and extrapulmonary, which can be thrombotic and nonthrombotic [7, 8]. Thrombotic causes can be associated with direct invasion of angiotensin-converting enzyme (ACE) receptors in the vascular endothelium, direct viral invasion of blood vessels or occlusion due to the formation of microthrombi, causing mesenteric ischemia (occlusal or neoclus). Non-thrombotic causes described in the literature include pancreatitis, peritonitis, bloating, and colitis and are associated with tropism of the virus to ACE receptors in the digestive tract $[7,8]$.

Here is our own observation of the case of casuistic development of peritonitis in a patient with COVID-19. 


\section{Hospital Sheet Chart}

Patient V., 55 years old, was treated in the surgical department of CI "Kryvyi Rih City Hospital 7" from 25.04.20 13h. $23 \mathrm{~min}$. to $26.04 .2010 \mathrm{~h}$. $36 \mathrm{~min}$. She was taken by an ambulance to the transfer from the city hospital №8 in Kryvyi Rih, where she was hospitalized. Complaints of intense abdominal pain, shortness of breath, general weakness.

According to the severity of the patient's condition, the anamnesis was clarified on the basis of the discharge epicrisis of the Kryvyi Rih City Hospital №8, and, in part, from the words of the patient's relatives. Complaints of general weakness, dizziness, headache, dry cough in the patient appeared about 10 days ago. Relatives of the patient denied contact with persons who had returned from abroad in the last 14 days (in the context of the WHO-declared pandemic COVID-19). Due to the progressive deterioration of the patient's condition (sharp general weakness, disturbances of consciousness, dizziness), relatives 18.04.20. called an ambulance, which took the patient to the Kryvyi Rih City Hospital №4, at the place of residence. There, on April 18, 20 , immediately upon admission, the patient was tested for antibodies to COVID-19, the result was negative, and then hospitalized in the therapeutic department with suspected encephalopathy of unknown origin.

Selective computed tomography (CT) of the brain and chest was performed. Conclusion of CT of the brain: "volume and focal pathology of the brain was not detected. No bone-destructive pathology was detected. CT-picture of Dyscirculatory encephalopathy (DEP) of 2 degrees". Conclusion of chest CT: "Tumor of the right breast. Secondary upper lobe pneumonia on the right". From 18.04.20 to 20.04.20 the patient was in the therapeutic department of the Kryvyi Rih City Hospital №4 with a diagnosis of "Tumor of the right breast, metastases to the chest, Dyscirculatory encephalopathy, cerebrospinal fluid, intoxication syndrome, sopor. Hypertension 2 degrees., risk 4 , heart failure 1 degree". Despite medical interventions, the patient's condition continued to progressively deteriorate, the level of consciousness - sopor, the phenomena of intoxication, respiratory failure progressed. For further treatment, on April 20, 20, the patient was transferred to the intensive care unit of Kryvyi Rih City Hospital №8. Chest radiography from 20.04.20: "on the right at the top of the area of inhomogeneous darkening of the lung tissue without clear contours, the roots of reduced structure, the sinuses are free. Pneumonia on the right in the presence of the clinic, in the absence - metastases on the right are not excluded". Electrocardiogram 20.04.20 - "sinus tachycardia, metabolic changes in the myocardium." 22.04.20p. performed CT of the chest, abdominal organs, pelvic organs, brain with intravenous amplification, the conclusion: "Bilateral pneumonia in the stage of reverse development. Signs of cardiomegaly, pulmonary hypertension. A simple cyst of the left kidney". The patient continued to receive intensive postsyndrome therapy, but - without improvement, the condition remained severe. 25.04.20p. the patient developed excruciating abdominal pain. Ultrasound examination of the abdominal cavity, conclusion: "ultrasound - signs of stagnant gallbladder, chronic cholecystitis, chronic pyelonephritis (exacerbation)". The review roentgenogram of abdominal organs, the conclusion: "intestinal impassability" is executed. The patient was examined by a surgeon of the 8th hospital, after which, with suspicion of acute intestinal obstruction, peritonitis and suspected coronavirus disease SARS-CoV-2 COVID-19, complicated by pneumonia, she was transferred to the surgical department of Kryvyi Rih City Hospital №7 with suspicion of a new coronavirus infection COVID-19.

\subsection{Status Praesens Communis}

The general condition of the patient is extremely serious. Level of consciousness - moderate stun. The position is passive, lying down. Patient with malnutrition. The skin is pale with an earthy tinge. The mammary glands are symmetrical, the nipples are not involved, without discharge. In the lower-inner quadrant of the right breast-palpable small-lobed seal, about $5 \times 3 \times 4.5 \mathrm{~cm}$ in size, moderately mobile, inguinal lymph nodes are not enlarged. In the lungs, vesicular breathing with a hard tinge, weakened in the lower parts, there are wet rales of various calibers on both sides. Respiratory rate-22 / minute. Heart tones are muffled, frequent, heart rate - $96 /$ minute, blood pressure - 100/60 $\mathrm{mm}$ of mercury, $\mathrm{t}-36,7^{\circ} \mathrm{C}$.

\subsection{Status Localis}

The tongue is dry, covered with a white layer. The abdomen is bloated, does not take part in the act of breathing, tense "board-like". On palpation sharply painful throughout, deep palpation is not achievable, sharply positive symptom of Shchotkin-Blumberg. At percussion - tympanitis, hepatic dullness is saved, blunting in lateral flanks is noted. Intestinal motility is not audible, there is a "slap noise". There is no urine in the transurethral catheter.

General blood test (25.04.20.): Hb 142 grams / liter, Er $4.6 \times 10^{\wedge} 12$ / liter, Color index 0.92,

Leukocytes $40,4 \times 10^{\wedge} 9 /$ liter, young $6 \%$, myelocytes $1 \%$, eosinophils $1 \%$, rod-shaped $31 \%$, segmental $46 \%$, lymphocytes $8 \%$, monocytes $7 \%$, erythrocyte sedimentation rate $3 \mathrm{~mm} /$ hour.

Cyto-test for antibodies to HIV: antibodies to HIV were not detected.

General analysis of urine (25.04.20): specific gravity 1015, Leukocytes 4-5 in the field of view, Er unchanged 25-30 in the field of view, erythrocytes changed 2-3 in the field of view, protein 0.242 , ketone bodies weakly positive (1+), transitional epithelium 2-3 in the field of view, granular cylinders 1-2 in the field of view.

Biochemical analysis of blood (25.04.20.): Total bilirubin $12.65 \mathrm{mmol} /$ liter, direct $8.05 \mathrm{mmol} /$ liter, indirect $4.6 \mathrm{mmol}$ / liter, total protein $46.02 \mathrm{~g} /$ liter, amylase $36.0 \mathrm{mg} /$ hours, fibrinogen 4.2; Prothrombin index 90\%.

On the basis of complaints, anamnesis data and objective research, it is established. 


\subsection{Diagnosis}

Acute peritonitis of unknown etiology. Intoxication. Multiple organ failure syndrome.

Concomitant pathology: Suspicion of coronavirus disease SARS-CoV-2 COVID-19. Bilateral community-acquired pneumonia. Grade 1 respiratory failure. Chronic pyelonephritis in the acute stage, chronic renal failure of 3 degrees. Intoxication encephalopathy. Right breast cancer?

After medical preparation and stabilization of vital functions, 25.02 .20 at 16.50 the patient underwent surgery: Diagnostic laparotomy, during which in the abdominal cavity was found about $800 \mathrm{ml}$ of serous dull yellowish effusion with an unpleasant odor and single flakes of fibrin, the distribution is equal. cavity. Inflammatory and destructive changes in the abdominal organs that could cause the development of peritonitis are absent. Rehabilitation and drainage of the abdominal cavity were performed. It should be noted that according to the latest English-language surgical journals, laparotomy is preferred when operating on patients with COVID-19, as there is a greater risk of viral aeration during laparoscopy due to pneumoperitoneum. However, this topic continues to be discussed and the question of the method of surgical intervention remains open [9-15]. In the postoperative period, the patient continued intensive post-syndrome therapy in the intensive care unit. The patient was on prolonged artificial, hemodynamics unstable, kept on medication. Despite the ongoing treatment, the patient's condition remained extremely serious. Against the background of severe manifestations of multiorgan failure syndrome, intoxication, 26.04.20 at 10.06 there was a cessation of blood circulation, clinical death was diagnosed. Unsuccessful resuscitation measures were initiated. 26.04.20 at 10.36 biological death was stated.

27.04.2020 a pathological anatomical autopsy was performed.

28.04.20 Answer received: Polymerase chain reaction for SARS-CoV-2 COVID-19 virus from 25.04.20 - positive result. After reviewing the histological preparations of the deceased patient by the chief pathologist of the city and region, as well as at the Department of Pathological Anatomy of the Dnieper State Medical Academy, the final diagnosis was made:

Pathological diagnosis:

MAIN: Coronavirus infection 2019 (result of Polymerase chain reaction to SARS-CoV-2 COVID-19 virus from 25.04.20 positive) / Bilateral hemorrhagic total pneumonia.

Complications BASIC: Intoxication. Generalized microcirculation disorder with DIC syndrome - thrombi in small pulmonary vessels, splenic infarction, necrotic nephrosis, serous fibrinous peritonitis (operation 25.04.20 laparotomy, revision, rehabilitation, drainage of the abdominal cavity). Pulmonary edema. Venous plethora.

ACCOMPANYING: Chronic ischemic heart disease: small-focal cardiosclerosis. Fatty hepatosis. Acute hemorrhagic nephritis. Fibrous mastopathy.

\section{Conclusions for Discussion}

Analyzing this case, we can draw the following conclusions:

1) This peritonitis is not caused by acute inflammatorydestructive pathology of the abdominal organs;

2) Peritonitis developed against the background of an extremely severe course of the new coronavirus disease SARS-CoV-2 COVID-19 in its terminal stage and is its direct consequence.

3 ) The mechanism of abdominal syndrome in this case COVID-19 is both pulmonary in origin (according to pathological examination, the patient was diagnosed with bilateral hemorrhagic total pneumonia) and extrapulmonary $[1,2]$.

4) The extrapulmonary mechanism of development of this COVID-19 - associated peritonitis is probably due to thrombotic causes that can be associated with direct viral invasion of ACE receptors into the vascular endothelium, direct viral invasion of blood vessels and occlusion as a result of microthrombus thrombosis. pulmonary vessels, splenic infarction, necrotic nephrosis) [1, 2, 7, 8].

This case is an atypical and unique variant of the new coronavirus disease SARS-CoV-2 COVID-19.

\section{References}

[1] Huang C, Wang Y, Li X, Ren L, Zhao J, Hu Y, et al. Clinical features of patients infected with 2019 novel coronavirus in Wuhan, China. Lancet. 2020; 395: 497-506. https://doi.org/10.1016/s0140-6736(20)30183-5

[2] R. Blanco-Colino, R. Vilallonga, R. Martin, et al. Suspected acute abdomen as an extrapulmonary manifestation of COVID-19 infection/ Gir Esp, 98 (May (5)) (2020), pp. 295296.

[3] A. O. E. Ahmed, M. Badawi, K. Ahmed, et al. Case report: COVID-19 masquerading as an acute surgical abdomen (2020). June 9; tpmd200559.

[4] Paula H. Takegawa, Marcia Calavaro Silva, Caroline Belluco, et al. "Inflammatory peritonitis in a child with COVID-19", Jornal of Pediatric Surgery Case Reports, Decenber 2021, 102077| https://doi.org/10.1016/j.epsc.2021.102077

[5] Ashraf Omer Elamin Ahmed, Sars F. Mohamed, Ahmed O. Saleh et al. Acute abdomen-like-presentation assotiated with SARS-CoV-2 infektion, IDCases 2020, e00895, ScienceDirekt| https//doi.org/10.1016/j.idcr.2020.e00895

[6] Al Argan, Safi G Algatari, Abir $\mathrm{H}$ Al Said et al. Gastrointestinal perforation secondary to COVID-19: case reports and literature review. Medicine (Baltimore). 2021 May 14; $100 \quad$ (19): $\quad$ e25771/ doi: 10.1097/MD.0000000000025771.

[7] Dragos Serban, Bogdan Socea, Cristinel Dumitru Badiu, et al. Acute surgical abdomen during the COVID-19 pandemie: Clinical and therapeutic challenges, Experimental and Therapeutic Medicine, March 22, 2021 https://doi.org/10.3892/etm.2021.9950 
[8] Islam H. Elrobaa and Karl I. New. COVID-19: Pulmonary Manifestations, Frontiers in Public Health, 28 september 2021| httrs://doi.org/10.3389/fpubh,2021.711616

[9] Di Saverio S., Khan M., Pata F., Ietto G., De Simone B., Zani E. Laparoscopy at all costs? Not now during COVID-19 and not for acute care surgery and emergency colorectal surgery: a practical algorithm from a Hub Tertiary teaching hospital in Northern Lombardy, Italy. J Trauma Acute Care Surg. 2020; 88 (6): 715-718. doi: 10.1097/TA.0000000000002727.

[10] De Simone B., Chouillard E., Di Saverio S., Pagani L., Sartelli M., Biffl W. L., Campanile F. C. Emergency surgery during the COVID-19 pandemic: what you need to know for practice. Ann R Coll Surg Engl. 2020: 1-10. 0.

[11] Zheng M. H., Boni L., Fingerhut A. Minimally invasive surgery and the novel coronavirus outbreak: lessons learned in
China and Italy. Ann Surg. 2020; 272 (1): e5-e6. doi: 10.1097/SLA.0000000000003924.

[12] Wang Juan, Du Guoqiang. COVID-19 may transmit through aerosol. Ir J Med Sci. 2020: 1-2.

[13] Champault G., Taffinder N., Ziol M., Riskalla H., Catheline J. M. C. Cells are present in the smoke created during laparoscopic surgery. Br J Surg. 1997; 84 (7): 993-995.

[14] Al-Balas M., Al-Balas H. I., Al-Balas H. Surgery during the COVID-19 pandemic: a comprehensive overview and perioperative care. Am J Surg. 2020 April 18 doi: 10.1016/j.amjsurg.2020.04018.

[15] Francis N., Dort J., Cho E., Feldman L., Keller D., Lim R., Wasco K. SAGES and EAES recommendations for minimally invasive surgery during COVID-19 pandemic. Surg Endosc. 2020: $1-5$. 